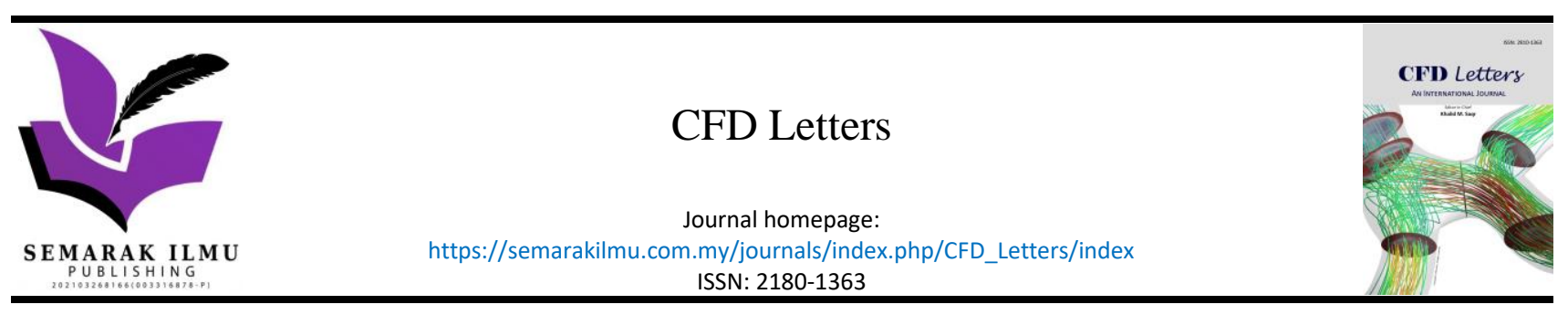

\title{
Optimization of inverse-Prandtl of Dissipation in standard $k-\varepsilon$ Turbulence Model for Predicting Flow Field of Crossflow Turbine
}

\author{
Candra Damis Widiawaty ${ }^{1,2}$, Ahmad Indra Siswantara ${ }^{1,{ }^{*}}$, Gun Gun R Gunadi ${ }^{1,2}$, Mohamad Arif \\ Andira $^{3}$, Budiarso ${ }^{1}$, Muhammad Arif Budiyanto ${ }^{1}$, M. Hilman Gumelar Syafei ${ }^{1}$, Dendy Adanta ${ }^{4}$

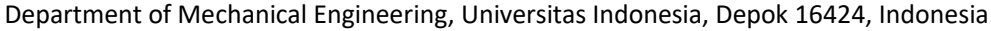

ARTICLE INFO

\section{Article history:}

Received 3 September 2021

Received in revised form 16 December 2021

Accepted 17 December 2021

Available online 7 January 202

\section{Keywords:}

Computational fluid dynamics; turbulent flows; turbulence modeling; Reynoldsaveraged Navier-stokes; crossflow water turbine; $k-\varepsilon$ turbulence model

\section{ABSTRACT}

Despite the successful use of the Standard $k-\varepsilon$ model in simulating turbulent flow for many industrially relevant flows, the model is still less accurate for a range of important problems, such as unconfined flows, curved boundary layers, rotating flows, and recirculating flows. As part of the authors' effort to extend the model applicability and reliability, this paper aims to study the effects of diffusivity parameter called the turbulent Prandtl number of dissipation rate $\left(\sigma_{\varepsilon}\right)$ on the Standard $k-\varepsilon$ model performance for predicting recirculating flow in a crossflow water turbine. The value of this parameter was varied from 0.5 to 1.5 in the CFD simulations, and the results were compared to the more sophisticated $k-\varepsilon$ model, namely the RNG $k-\varepsilon$, which has been first qualitatively validated by an experimental result. In addition, the parameter value was also adjusted using the Multi-Linear Regression (MLR) method ranging from 0.42 to 1.5 to complement the CFD simulations. It was observed that reducing the $\sigma_{\varepsilon}$ value is effective in minimizing the average deviation of the turbulence properties concerning the RNG $k-\varepsilon$ model. However, the adjusted $k-\varepsilon$ model still faces difficulty in accurately predicting the pressure and velocity field. Based on this result, adjusting the $\sigma_{\varepsilon}$ constant in the Standard $k-\varepsilon$ turbulence model has the potential to improve the model performance for modelling recirculating flow in terms of the turbulence properties, but still needs further investigation for the flow properties.

\section{Introduction}

Turbulent flow is commonly accompanied by the flow which experiences reversal, called recirculating flow, frequently caused by the separation of a boundary layer. Unlike attached-flow turbulence, recirculating flow is governed by large-scale, outer region eddies, which urge a wake-like pattern on the flow [1]. One of the examples of the importance of recirculating flow in engineering practice is the crossflow water turbine. Presently, the massive use of crossflow water turbine has

\footnotetext{
* Corresponding author.

E-mail address: a_indra@eng.ui.ac.id (Ahmad Indra Siswantara)
} 
been restricted due to its lower efficiency compared to other types of turbines, which can achieve efficiencies near to $90 \%$ [2]. This problem is heavily associated with the recirculation phenomenon. The recirculating flow which occurred in the middle region of the runner will absorb the mean flow energy and thus reduce the kinetic energy transfer to mechanical energy on the turbine blades, causing a drop in the system efficiency. Being able to accurately predict such phenomena is crucial to improving the efficiency of engineering equipment.

Computational fluid dynamics (CFD) with the Reynolds-Averaged Navier-Stokes (RANS) equations approach has been widely used to study fluid flow involving turbulence due to its computational efficiency since the method only focuses on the mean flow and the effects of turbulence on mean flow properties. In essence, the RANS equations are derived through the Reynolds-averaging process of the Navier-Stokes equations, resulting in six extra terms called Reynolds stresses, which need to be modeled to close the equations [3]. There are two common approaches to model these stresses; Reynolds Stress Model (RSM) and the Boussinesq eddy-viscosity hypothesis. The use of RSM is less frequent because of its high computational cost, while the latter model already provides good accuracy with moderate computational power [4]. In the Boussinesq eddy-viscosity hypothesis, the Standard $k-\varepsilon$ and RNG $k-\varepsilon$ models are generally used since the results have been thoroughly proven to be used for technical analysis in the industry [5].

In the case of recirculating flow, the RNG $k-\varepsilon$ outperforms the standard model because it contains more complex models, including the additions of the swirl effect term of turbulence, the differential formula for effective viscosity, the analytical formula for the turbulent Prandtl number, and the enhancement in the generation and destruction terms in the turbulent dissipation rate equation $(\varepsilon)[6,7]$. Yakhot et al., [8] proved the ability of the RNG $k-\varepsilon$ model in accurately predicting recirculating flow over a backward-facing step, which gave rise to considerable attentiveness to employ the model into commercial CFD codes. Nonetheless, [9]reported that the RNG $k-\varepsilon$ model is only sensitive to the magnitude of the strain parameter magnitude $\eta$ and not to its sign. As a result, the model will give the same effect if the geometry is contracting or expanding, which is not true in reality. In addition to this sensitivity issue, the computational cost of the RNG $k-\varepsilon$ is also higher than the standard model [4].

On the other hand, the numerical studies of the Standard $k-\varepsilon$ model conducted by some authors [10-18] showed relatively good results and robustness but the model is still less accurate in predicting the flow details, especially in rotating, recirculating, and large separation flow. The Standard $k-\varepsilon$ model tends to cause higher turbulent kinetic energy, turbulent dissipation, and turbulent viscosity than the actual conditions. This is suspected due to the turbulent Prandt number in the $\varepsilon$ equation which is assumed to be unity $\left(\sigma_{\varepsilon}=1\right)$ [19-22]. The early attempt of simulating recirculating flow in a backward-facing step configuration using the Standard $k-\varepsilon$ model [23] indicated that the model underpredicted the reattachment point by the order of $20-25 \%$ compared to the experimental results. Avva et al., [24] addressed this problem by suggesting that the underpredicted value was caused by the insufficient computational grid resolution. When the finer grid was used, they observed that the error was only of the order of $10 \%$. Afterward, there has been significant number of studies that tried to improve the Standard $k-\varepsilon$ model performance, such as modifications to account for streamline curvature based on the algebraic stress model [25], performing calculations with a multiple scale $k-\varepsilon$ [26], and development of the anisotropic model [27], to name but a few. However, no detailed study to investigate the effects of the $\sigma_{\varepsilon}$ change on the model performance in predicting recirculating flow, particularly for a crossflow water turbine.

A preliminary effort to fill this gap has been carried out by the authors by proposing $\sigma_{\varepsilon}$ values greater than 1 [28], which concluded that the small change of $\sigma_{\varepsilon}$ can be very sensitive to the results. There was a considerable difference on the turbulent dissipation rate value between the $\sigma_{\varepsilon}$ value of 
1.4 and 1.5 , while the values of 1.1 to 1.4 exhibited a similar result. Hence, this research aims to further investigate the effect of changing the turbulent Prandtl number in the $\varepsilon$ equation on the flow and turbulence properties in recirculation flow phenomena within a broader range of $\sigma_{\varepsilon}$ values, which might improve the Standard $k-\varepsilon$ model performance in providing better accuracy with low computational cost. The study was carried by simulating the flow inside a crossflow water turbine using the Standard $k-\varepsilon$ turbulence model and the $\sigma_{\varepsilon}$ parameter value was proposed to be varied from 0.5 to 1.5. The assessment of the standard model performance was done with respect to the RNG $k-\varepsilon$ model, which has been first validated qualitatively by an experimental result using particle image velocimetry (PIV) technique. The performance of the standard model is said to be good if it can minimize the deviations of pressure, velocity, and turbulence properties from the results obtained using the RNG model. Furthermore, the parameter adjustments were also performed with the value ranging from 0.42 to 1.5 using the Multi-Linear Regression (MLR) method to verify and complement the CFD analysis.

\section{Mathematical Formulations of the $\boldsymbol{k}-\boldsymbol{\varepsilon}$ Turbulence Models}

Recall the Reynolds-Averaged Navier-Stokes (RANS) equations for an unsteady incompressible Newtonian fluid in Einstein notation in Cartesian coordinates as it is the underlying mathematical model for the present numerical simulation.

$\rho\left(\frac{\partial \bar{u}_{i}}{\partial t}+\bar{u}_{j} \frac{\partial \bar{u}_{i}}{\partial x_{j}}=\frac{\partial}{\partial x_{j}}\left[-\bar{p} \delta_{i j}+\mu\left(\frac{\partial \bar{u}_{i}}{\partial x_{j}}+\frac{\partial \bar{u}_{j}}{\partial x_{i}}\right)-\rho \overline{u^{\prime}{ }_{\imath}^{\prime}{ }_{j}^{\prime}}\right]+\rho f_{i}\right.$

The equations contain six additional extra terms $\rho \overline{u^{\prime}{ }_{l}^{\prime}{ }_{J}^{\prime}}$ called the Reynolds stresses, which make the number of unknowns is more than the number of equations. Using the Boussinesq eddy-viscosity hypothesis, the Reynolds stresses can be represented with the mean flow velocities,

$\rho \overline{u^{\prime}{ }_{l} u^{\prime}{ }_{j}}=\mu_{t}\left(\frac{\partial \bar{u}_{i}}{\partial x_{j}}+\frac{\partial \bar{u}_{j}}{\partial x_{i}}\right)-\frac{2}{3} \rho k \delta_{i j}$

where $k=\frac{1}{2}\left(\overline{u^{\prime 2}}+\overline{v^{\prime 2}}+\overline{w^{\prime 2}}\right)$ is the turbulent kinetic energy per unit mass, $\delta_{i j}$ is the Kronecker delta $\left(\delta_{i j}=1\right.$ if $i=j$ and $\delta_{i j}=0$ if $i \neq j$ ), and $\mu_{t}$ is the turbulent or eddy viscosity. Thus, if the $k$ and $\mu_{t}$ are known, the RANS equations are closed and can be solved to find the mean value of flow quantities. It is therefore the main objective of the $k-\varepsilon$ turbulence models-which consist of two transport equations for the turbulent kinetic energy $k$ and its dissipation rate $\varepsilon$-to calculate the turbulent kinetic energy and express the eddy viscosity $\mu_{t}$ as a function of $k$ and $\varepsilon$.

$\mu_{t}=\rho C_{\mu} \frac{k^{2}}{\varepsilon}$

The Standard $k-\varepsilon$ model is a semi-empirical model proposed by Launder and Spalding [19] which was derived from the assumption that the flow is fully turbulent, and the effects of molecular viscosity are neglected. The turbulent kinetic energy equation is written as

$\frac{\partial(\rho k)}{\partial t}+\frac{\partial\left(\rho k \overline{u_{l}}\right)}{\partial x_{i}}=\frac{\partial}{\partial x_{j}}\left[\frac{\mu_{t}}{\sigma_{k}} \frac{\partial k}{\partial x_{j}}\right]+2 \mu_{t} S_{i j} S_{i j}-\rho \varepsilon$

and for the dissipation rate of the turbulent kinetic energy 
$\frac{\partial(\rho \varepsilon)}{\partial t}+\frac{\partial\left(\rho \varepsilon \overline{u_{l}}\right)}{\partial x_{i}}=\frac{\partial}{\partial x_{j}}\left[\frac{\mu_{t}}{\sigma_{\varepsilon}} \frac{\partial \varepsilon}{\partial x_{j}}\right]+C_{1 \varepsilon} \frac{\varepsilon}{k} 2 \mu_{t} S_{i j} S_{i j}-C_{2 \varepsilon} \rho \frac{\varepsilon^{2}}{k}$

where $\bar{u}_{i}$ is the mean velocity components, $S_{i j}$ represent the component of deformation rate (mean velocity gradients), $\sigma_{k}$ and $\sigma_{\varepsilon}$ are the turbulent Prandtl numbers for $k$ and $\varepsilon$, respectively. The default value for all constants have been determined from experiments for a variety of turbulent flows and are defined as follows; $C_{\mu}=0.09, C_{1 \varepsilon}=1.44, C_{2 \varepsilon}=1.92, \sigma_{k}=1.30, \sigma_{\varepsilon}=1.00$. The focus of the present study is on the $\sigma_{\varepsilon}$ constant, which is the diffusivity parameter for the turbulent dissipation rate. From the $\varepsilon$ equation above, it can be seen that decreasing the $\sigma_{\varepsilon}$ value will increase the overall diffusivity parameter value, and thus enhance the diffusion process.

The RNG $k-\varepsilon$ model, on the other hand, was derived using statistical technique called the renormalization group (RNG) devised by Yakhot and Orszag of Princeton University. The equations for the RNG $k-\varepsilon$ model are written in the following form

$\frac{\partial(\rho k)}{\partial t}+\frac{\partial\left(\rho k \overline{u_{l}}\right)}{\partial x_{i}}=\frac{\partial}{\partial x_{j}}\left[\alpha_{k} \mu_{e f f} \frac{\partial k}{\partial x_{j}}\right]+2 \mu_{t} S_{i j} S_{i j}-\rho \varepsilon$

$\frac{\partial(\rho \varepsilon)}{\partial t}+\frac{\partial\left(\rho \varepsilon \overline{u_{l}}\right)}{\partial x_{i}}=\frac{\partial}{\partial x_{j}}\left[\alpha_{\varepsilon} \mu_{e f f} \frac{\partial \varepsilon}{\partial x_{j}}\right]+C_{1 \varepsilon} \frac{\varepsilon}{k} 2 \mu_{t} S_{i j} S_{i j}-C^{*}{ }_{2 \varepsilon} \rho \frac{\varepsilon^{2}}{k}$

where

$\mu_{e f f}=\mu+\mu_{t}$

and $\mu_{t}$ is commonly computed using Eq. (3) for high-Reynolds number, while to handle the lowReynolds number and near-wall flows the RNG model provides a differential equation for calculating the turbulent viscosity [29]. For the RNG model, the constants are as follows

$C_{\mu}=0.0845, C_{1 \varepsilon}=1.42, C_{2 \varepsilon}=1.68, \alpha_{k}=\alpha_{\varepsilon}=1.393$

$\alpha_{k}$ and $\alpha_{\varepsilon}$ are called the inverse Prandtl number for $k$ and $\varepsilon$ respectively, which represent the effects of small-scale turbulence in the diffusion terms. It can be observed that the major difference between the Standard and RNG $k-\varepsilon$ model resides in the $\varepsilon$ equation, which significantly enhances the accuracy of the model for rapidly strained and swirling flows, since

$C_{2 \varepsilon}^{*}=C_{2 \varepsilon}+\frac{C_{\mu} \eta^{3}\left(1-\eta / \eta_{0}\right)}{1+\beta \eta^{3}}$

and

$\eta=\frac{k}{\varepsilon} \sqrt{2 S_{i j} S_{i j}} \eta_{0}=4.38 \beta=0.012$

There are two scenarios of restrained flow that can be analyzed to assess the behavior of the RNG $k-\varepsilon$ model. In the case of low to moderate strained flows $\left(\eta<\eta_{0}\right)$, such as the logarithmic layer, the value of $C_{2 \varepsilon}^{*}$ is larger than $C_{2 \varepsilon}$ which causes the large destruction of dissipation rate, so that the RNG model is less dissipative than the standard model. On the contrary, in regions near walls where large strain rate occurs $\left(\eta>\eta_{0}\right)$, the value of $\varepsilon$ will be higher (more dissipative) due to the lower 
destruction term and consequently, the kinetic energy will be reduced, resulting in a lower turbulent viscosity than the Standard $k-\varepsilon$ model.

\section{Methodology}

\subsection{Experimental Investigation Using Particle Image Velocimetry (PIV) Technique}

An experiment to visualize the flow field inside a crossflow water turbine was carried out to provide a qualitative validation reference for the CFD simulations. The experiment leveraged Crossflow Water Turbine and Dantec PIV system facilities in the University of Indonesia (UI) Fluid Mechanics Laboratory. Figure 1 displays the experimental setup to obtain the flow field visualization inside the crossflow water turbine using PIV technique. In the first experiment, the crossflow turbine was run in steady-state condition and the snapshots of the flow were taken using a high-speed camera embedded in the PIV system. Unfortunately, the result of the PIV for the dynamics experiments of the crossflow turbine gives a quite high uncertainty an inaccurate result. Therefore, the crossflow turbine is locked and maintained in static condition to give a more accurate and acceptable experiment result.

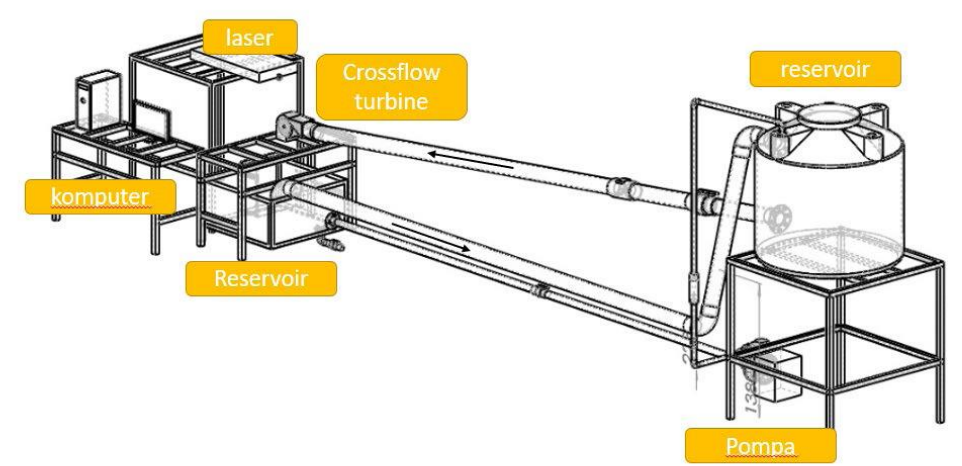

Fig. 1. Experimental setup of the crossflow water turbine using Dantec PIV system in Fluid Mechanics Laboratory of Universitas Indonesia

To obtain the flow visualization, the PIV system required seeding particles that were collected inside the reservoir as depicted in Figure 2. The particles must first be blended with the water before they were injected into the turbine. Consecutively, a test was performed to ensure the homogeneity of the particle-water mixture, meaning that the particles were spread uniformly inside the water. Once the homogeneity was achieved, the energy and focus of the camera lens were adjusted by manually rotating the knob and laser control panel until the particles could be properly captured by the camera.

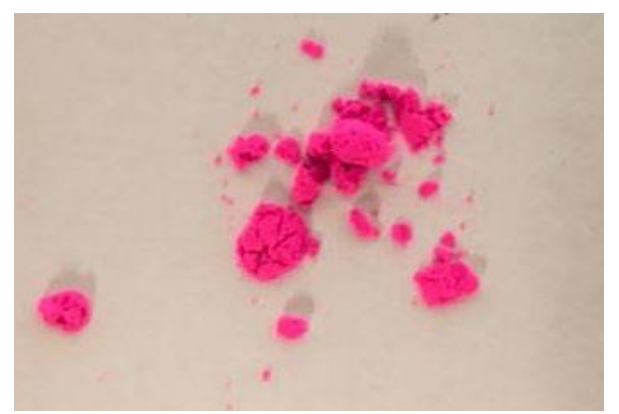

Fig. 2. Seeding particles used in the experiment 
In the PIV system, picture calibration is highly important at getting the scaling factor (pixels $/ \mathrm{mm}$ ). This factor was obtained by measuring the reference distance in the location of interest of the crossflow turbine. The estimated distance was then sent as an input to the PIV software. It was obtained that the scaling factor is approximately 6 pixels $/ \mathrm{mm}$. Finally, the PIV was calibrated to correctly measure the flow rate by comparing the measured velocity value with the computed velocity value (measured volume flow rate/turbine inlet area). The result was that the average velocity from the PIV measurement was $1.147 \mathrm{~m} / \mathrm{s}$ and the computed velocity was $1.439 \mathrm{~m} / \mathrm{s}$, which showed that the uncertainty was under $3 \%$ and thus acceptable to be used for the experiment.

\subsection{Modeling of Turbulent Recirculating Flow in a Crossflow Water Turbine}

The computational modeling of a crossflow water turbine was performed using CFDSOF $^{\circledR}$ software by CCIT Group Indonesia. CFDSOF consists of CFD methods basic such as mass conservation, momentum conservation, and energy conservation, where these equations were solved by finite volume method. This software was developed by the AIR group research team which offer turbulence model, heat transfer simulation (conduction, convection, and radiation), combustion, multiphase, and others. It also has been used by several researchers with good simulation results. Moreover, researchers are familiar with the appearance of this software, making it easier to do simulations [28,30-32]. The main simulation objective is to study the effects of changing the $\sigma_{\varepsilon}$ value from 0.5 to 1.5 on the recirculating flow prediction performance using the Standard $k-\varepsilon$ turbulence model. The best performance is defined as the model with a certain $\sigma_{\varepsilon}$ value which gives the minimum deviations for the RNG $k-\varepsilon$ model in terms of flow and turbulence properties, such as velocity, pressure, turbulent kinetic energy, rate of dissipation of turbulent kinetic energy, and turbulent effective viscosity.

\subsubsection{Geometry of the model}

The three-dimensional crossflow turbine domain and configuration used in the simulation are shown in Figure 3 (dimensions in $\mathrm{mm}$ ).

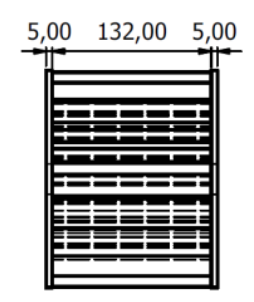

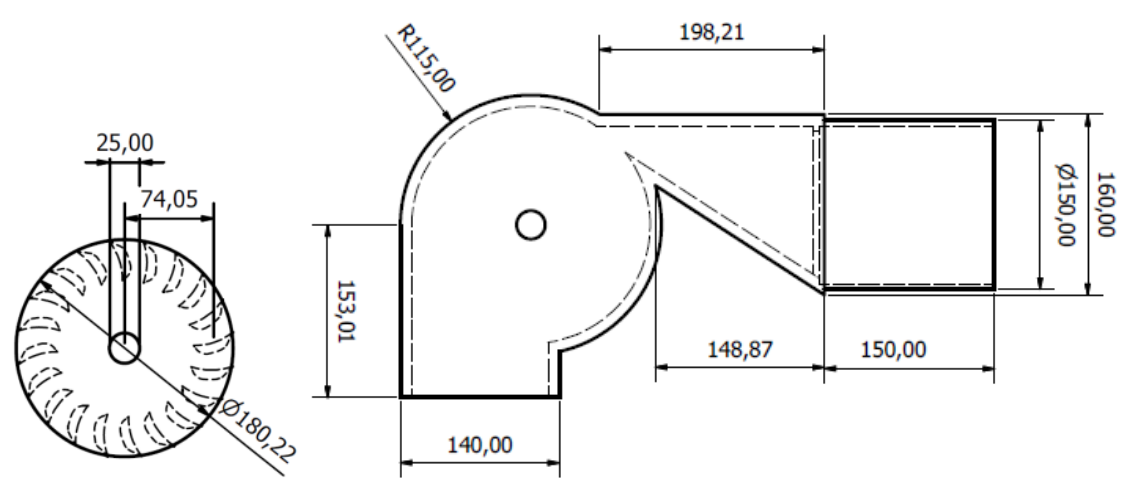

Fig. 3. Crossflow turbine geometry

\subsubsection{Mesh generation}

The mesh consists of structured three-dimensional (hexahedral) cells as visualized in Figure 4 . To ensure the resulting mesh has been sufficient enough to deliver computational results with minimum numerical errors due to the domain discretization process, a mesh independency test was carried out for three different mesh configurations. The Grid Convergence Index (GCI) was used to determine 
the independence of the mesh configuration and thus can select the optimum mesh in terms of computational accuracy and cost. $\mathrm{GCl}$ to get the medium mesh is

$G C I_{12}=F_{S} \times\left|\frac{1}{\tau_{\text {fine }}} \frac{\tau_{\text {medium }}-\tau_{\text {fine }}}{r_{12}{ }^{P n}-1}\right| \times 100 \%$

The value of $F$ is 1.25 , where $F$ is a safety factor. Analysis of the convergence observed $\left(p_{n}\right)$ was carried out using Eq. (13). Richardson's extrapolation $\left(p_{r h}=0\right)$ was applied for fine to medium mesh category, to get an estimated value of velocity recovery at zero grid distance using Eq. (15) [33]

$p_{n+1}=\ln \left[\left(\frac{\tau_{\text {coarse }}-\tau_{\text {medium }}}{\tau_{\text {medium }}-\tau_{\text {fine }}}\left(r_{12}^{\text {Pn }}-1\right)\right)+r_{12}{ }^{p n}\right] / \ln \left(r_{12} \cdot r_{23}\right)$

$P r_{h=0}=\tau_{\text {fine }}-\left(\frac{\tau_{\text {medium }}-\tau_{\text {fine }}}{r_{12}{ }^{\text {Pn+1 }}-1}\right)$

$r_{12}=\left(\frac{\tau_{\text {medium }}}{\tau_{\text {fine }}}\right)^{0.5}$

In this case, the repair ratio is given by a symbol $r_{12}$, while the mesh number is given the symbol $M$. The grid independence test was performed using the RNG $k-\varepsilon$ model and the mesh configurations along with their respective $\mathrm{GCl}$ percentage are shown in Table 1 below. Based on this result, the mesh with cell counts of $47508(214 \times 74 \times 3)$ is chosen for the CFD simulation.

Table 1

Mesh independency test result

\begin{tabular}{lllll}
\hline Number of mesh & $\tau(\mathrm{Nm})$ & $\mathrm{r}$ & $\mathrm{p}$ & $\mathrm{GCl}(\%)$ \\
\hline 42840 & 6.846 & & & \\
45156 & 6.495 & 1.026675 & 33.40257 & $4.79 \%$ \\
47508 & 6.484 & 1.025712 & 33.97506 & $0.15 \%$ \\
\hline
\end{tabular}

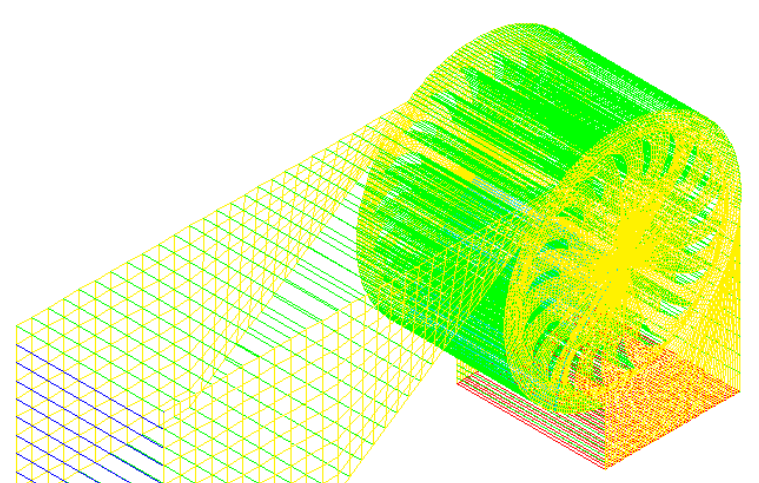

Fig. 4. Visualization of the 3D mesh 


\subsubsection{Simulation setup}

For the boundary conditions, the inlet condition is imposed by the normal velocity type with the value of $3.13 \mathrm{~m} / \mathrm{s}$. The angular velocity of the runner is $0 \mathrm{rad} / \mathrm{s}$ (static runner) to match the experimental condition and the pressure outlet is set at the outlet boundary. The working fluid is water at $250 \mathrm{C}\left(\rho=1000 \mathrm{~kg} / \mathrm{m}^{3}, \mu=8.68 \times 10^{-4} \mathrm{~kg} / \mathrm{m} . \mathrm{s}\right)$. Based on the previous study, $\Delta t=$ 0.0001 seconds is needed to represent changes that occur in the flow plane. The boundary condition setting in this simulation is set as follow: (i) The turbine runner is set on a static condition where the rotational velocity is zero, (ii) an inlet velocity is set in normal direction to the inlet surface with the value of $3.13 \mathrm{~m} / \mathrm{s}$, (iii) an outlet pressure boundary with atmospheric pressure condition is set at the outlet of the flow, (iv) the gravitational acceleration is considered.

\section{Results and Discussion}

Before proceeding any further into the analysis, the computational model was first validated qualitatively by the experimental result obtained by using the PIV technique in terms of velocity field. Based on Figure 5 below, the velocity field produced by the RNG $k-\varepsilon$ model exhibits identical recirculating flow patterns with the experimental result depicted in Figure 6 during the separation phenomenon on the first stage blade

In light of this result, the RNG $k-\varepsilon$ model is considered to be able to capture the prominent flow features and thus is used as a benchmark for assessing the Standard $k-\varepsilon$ model performance when varying the dissipation rate of turbulent kinetic energy parameter.

Figure 7 to Figure 10 present the simulation result using the RNG $k-\varepsilon$ model for flow and turbulence properties. The highest velocity occurs on the first stage blade inlet that causes the highest momentum transfer to the blade, as shown in Figure 7. Furthermore, there exists flow separation on the upper surface of several blades, which will negatively affect the ability of the water to move the neighboring blades. Outside the blade region, the water flows with higher velocity than the inner region following the circular curvature of the turbine case until it reaches the outlet duct and leaves the computational domain.

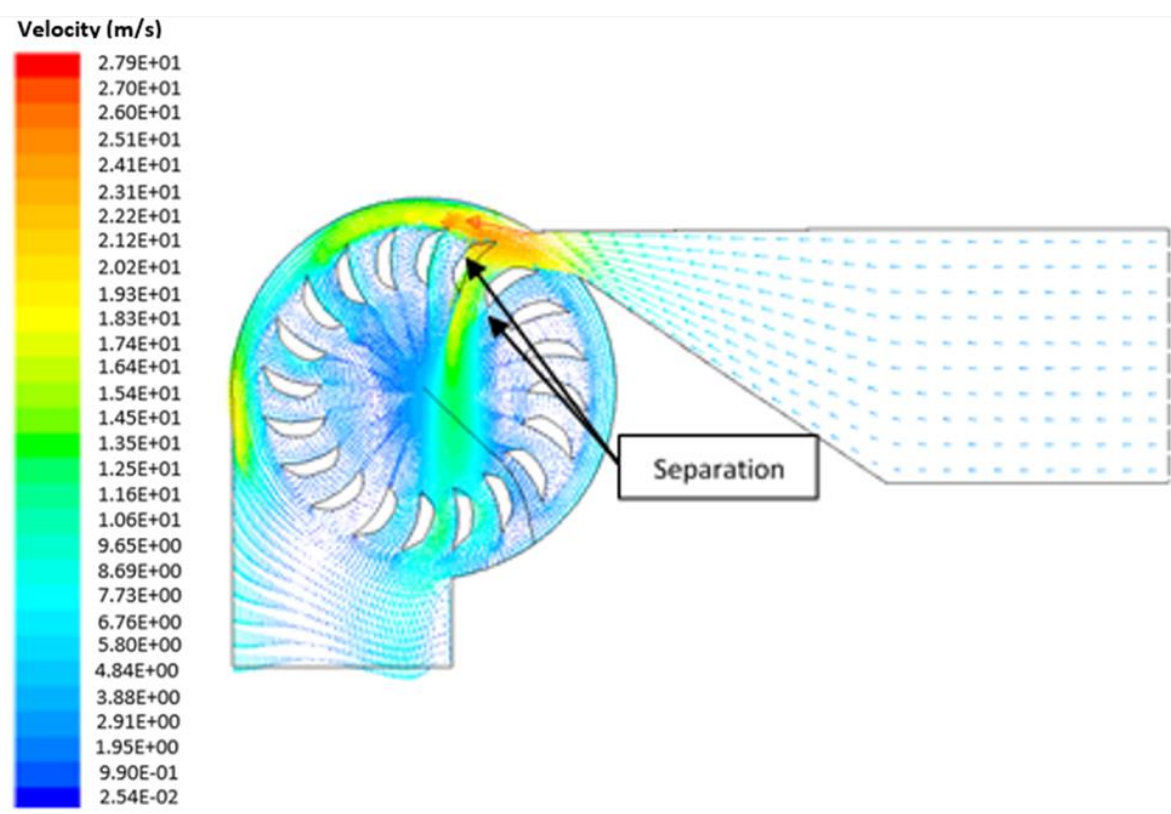

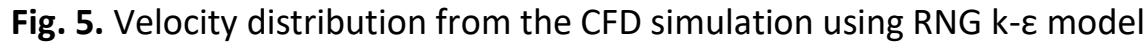



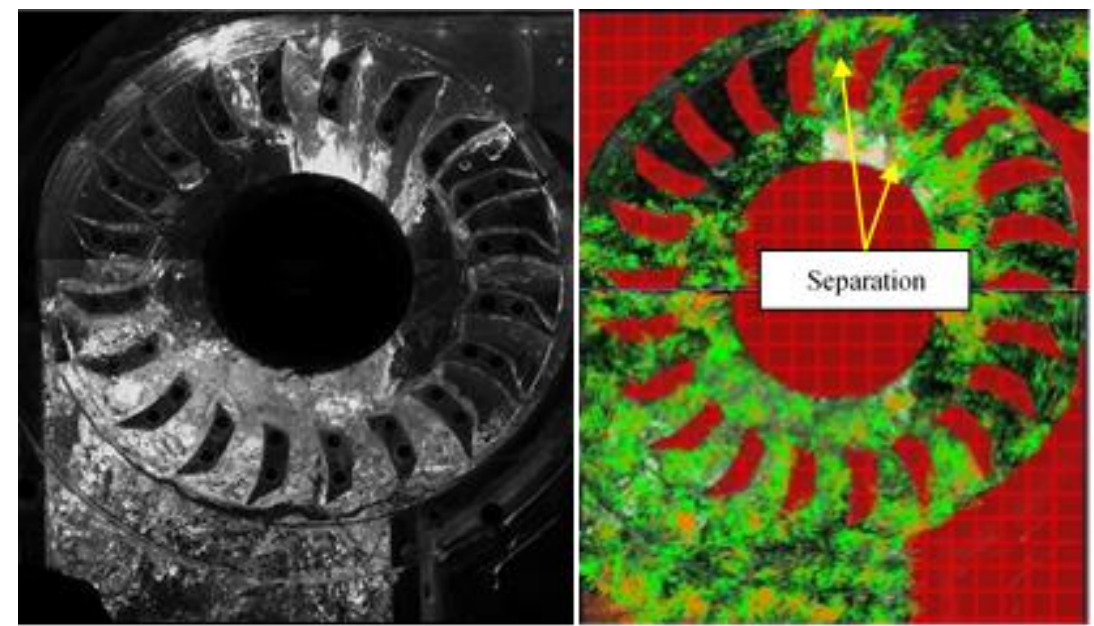

Fig. 6. Velocity distribution from the experiment using PIV

Figure 8 shows the distribution of absolute total pressure contour which can provide insights into energy associated with the flow. It can be observed that the total pressure has relatively uniform values in the nozzle, but then its values drop dramatically after entering the runner region. Moreover, the regions between blades contain lower total pressure regions than their surroundings, implying the presence of flow separation and recirculation phenomena, which absorb energy from the mean flow. The turbulent kinetic energy $(k)$ and the rate of dissipation of turbulent kinetic energy $(\varepsilon)$ contours are given in Figure 9 and Figure 10 respectively. The turbulent kinetic energy values near the first stage blade are large since the flows are being accelerated by the nozzle, resulting in greater velocity values. These large turbulent kinetic energy fields indicate the large size of eddies, while the other lower energy regions mean the eddies have got smaller in size through the energy cascade mechanism.

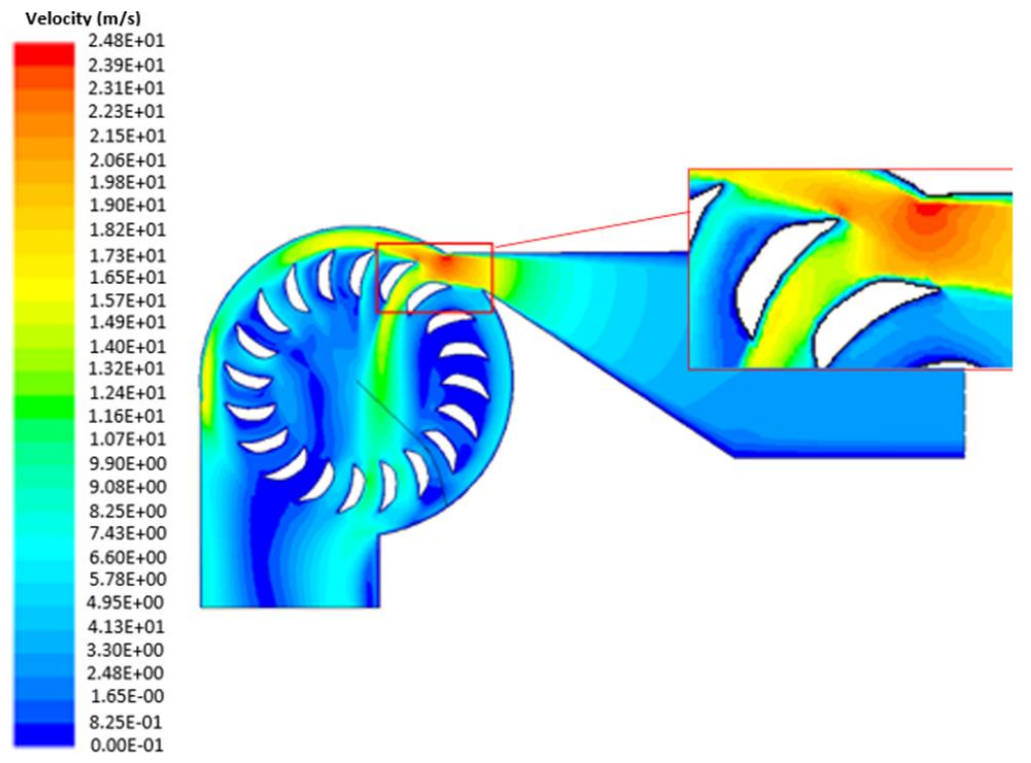

Fig. 7. Velocity field contour from the CFD simulation using RNG k- $\varepsilon$ model 


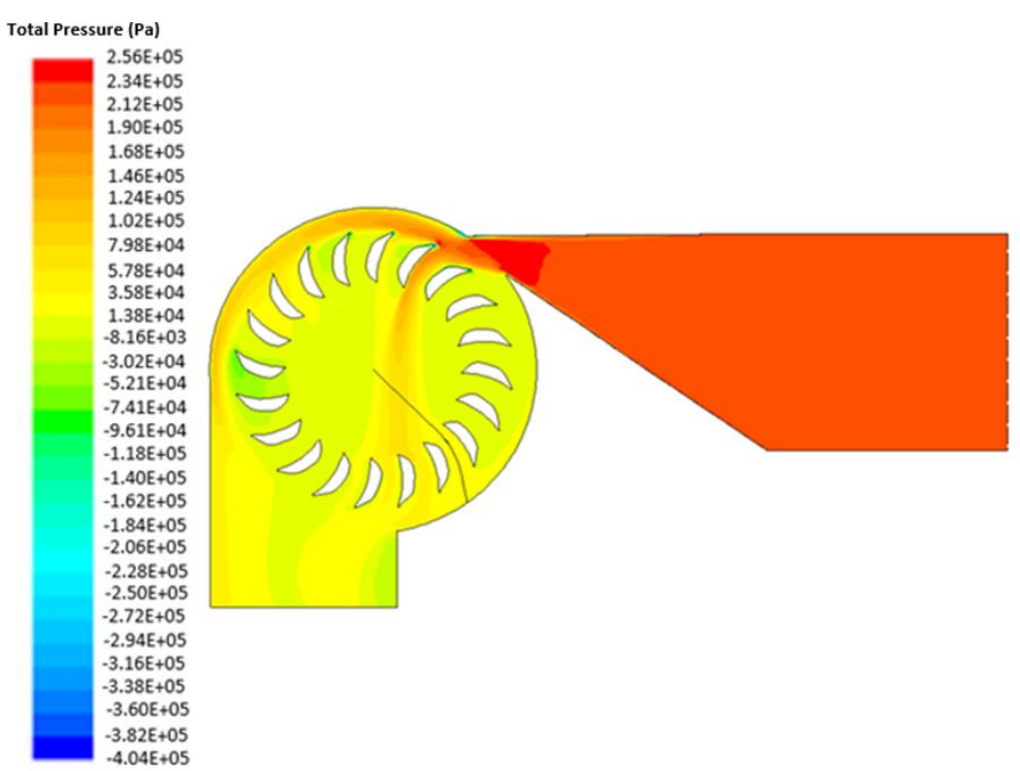

Fig. 8. Pressure field contour from the CFD simulation using RNG $k-\varepsilon$ model

There are some large turbulence dissipation rates in the first stage blade inlet area and other several turbine regions, particularly on the leading-edge section. These high dissipation rates arise when the flow velocities are relatively high and start to separate from the leading-edge surfaces. By correlating this information with the previous knowledge of turbulent kinetic energy, it can be concluded that the large turbulence dissipation rate occurs under large turbulent kinetic energy since the contour of turbulent dissipation rate shows significant values only in the area where the turbulent kinetic energy exhibits the contour colors of yellow to red. Over and above that, one can firmly remark that the large turbulent kinetic energy, as well as the large dissipation rate, are located at the first stage blade after the converging section of the nozzle. It is an important note for engineering perspective to minimize the energy dissipation due to the turbulence in these critical regions to improve the transfer of kinetic energy to mechanical energy on the blades.

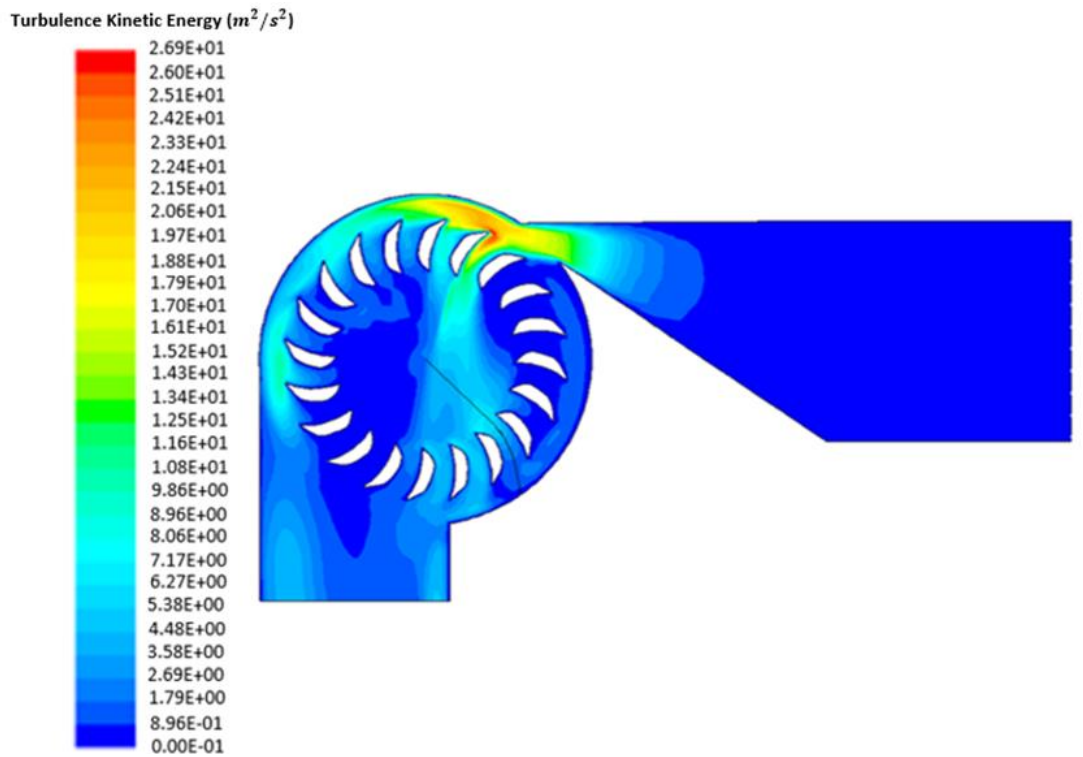

Fig. 9 Turbulent kinetic energy field contour from the CFD simulation using RNG k- $\varepsilon$ model 


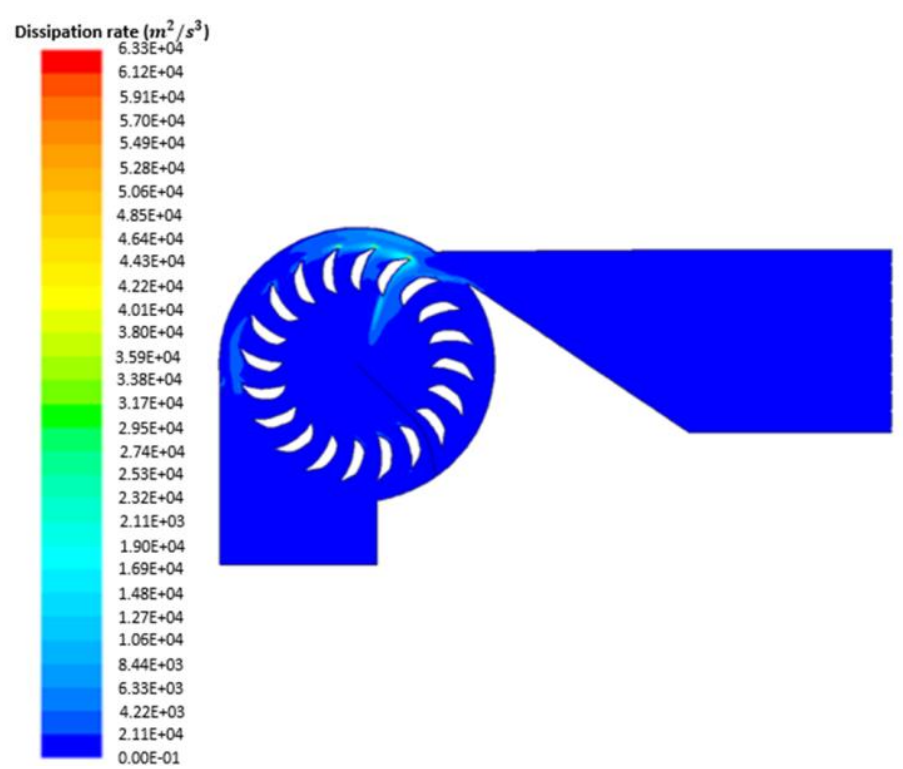

Fig. 10 Dissipation rate of turbulent kinetic energy field contour from the CFD simulation using RNG k- $\varepsilon$ model

As stated earlier, the focus of the present research is to study the influence of the $\sigma_{\varepsilon}$ value on the Standard $k-\varepsilon$ model performance in predicting recirculating flow. Since the critical region in which the recirculating flow phenomenon occurs is the area of the first stage blade turbine, the flow field and turbulence properties values will be assessed along the vertical line which spans from inlet to outlet channel of the first stage turbine. The results of varying turbulent Prandtl number of dissipation rate from 0.5 to 1.5 are given by Figure 11 to Figure 14.

It is convenient to start the discussion with the turbulence properties. There is a well-recognizable pattern that shows that the decreasing of the $\sigma_{\varepsilon}$ value will reduce the turbulent kinetic energy (Figure 13), the rate of dissipation of turbulent kinetic energy (Figure 14), and the turbulent effective viscosity (Figure 15). Furthermore, all the $\sigma_{\varepsilon}$ values exhibit an identical pattern, where the turbulence properties rise until $Y / Y_{\max }$ values of 0.3 or 0.4 and then decline across the remaining positions on the line. Based on these two graphics, the $\sigma_{\varepsilon}$ value of 0.5 gives the minimum deviations

with respect to the RNG $k-\varepsilon$ model. This is an intriguing founding since the author's previous research showed a contrast result [28]. It was found that for the recirculating flow simulation inside a crossflow turbine, increasing the $\sigma_{\varepsilon}$ value will reduce the turbulent kinetic energy and its dissipation rate and thus the optimum value of $\sigma_{\varepsilon}$ was found to be 1.5. The noticeable differences are the previous research used air as the fluid and the runner was set to have an angular velocity, while in this current study the fluid is water and the runner is considered to be static, to match the PIV measurement.

Nonetheless, the case is different from the total pressure (Figure 11) and velocity (Figure 12) fields. In the pressure graphic, a similar pattern is observed regarding the declining value of total pressure as the $\sigma_{\varepsilon}$ decreases, but the minimum deviation is found when the $\sigma_{\varepsilon}$ value is 1.3 , which is in contrast with the turbulence properties results. For the velocity field, changing $\sigma_{\varepsilon}$ does not give a significant effect to the velocity values. At location $Y / Y_{\max }=0.5$, all velocity values almost coincide each other, while at $Y / Y_{\max }=1.0$, the velocity predicted by the Standard $k-\varepsilon$ model failed to accurately approach the value of the RNG $k-\varepsilon$ model. 


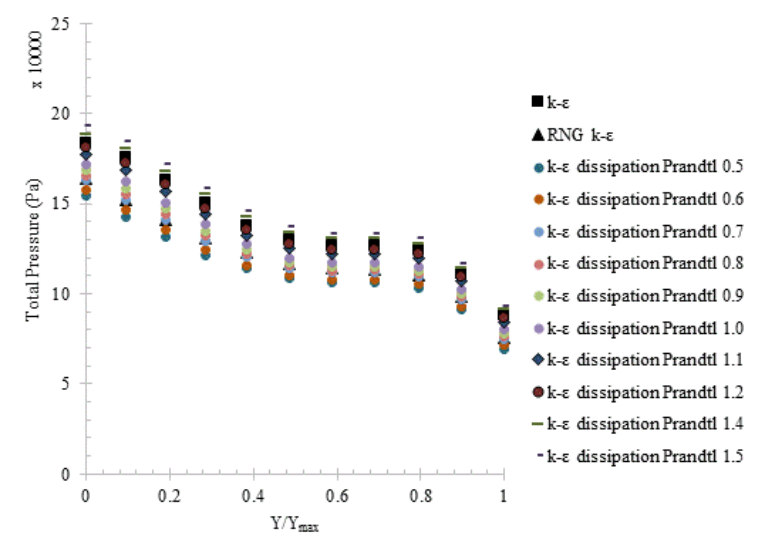

Fig. 11. Total pressure distribution along the $y$ axis of the crossflow turbine blade

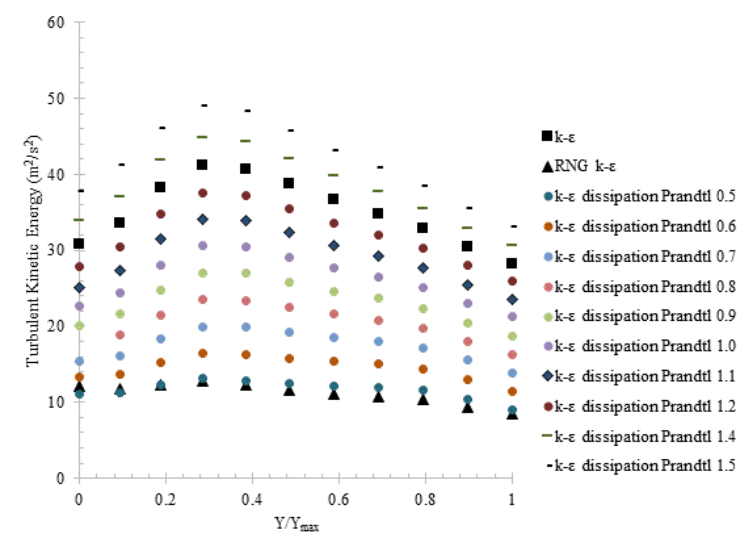

Fig. 13. Turbulent kinetic energy distribution along the $y$-axis of the crossflow turbine blade

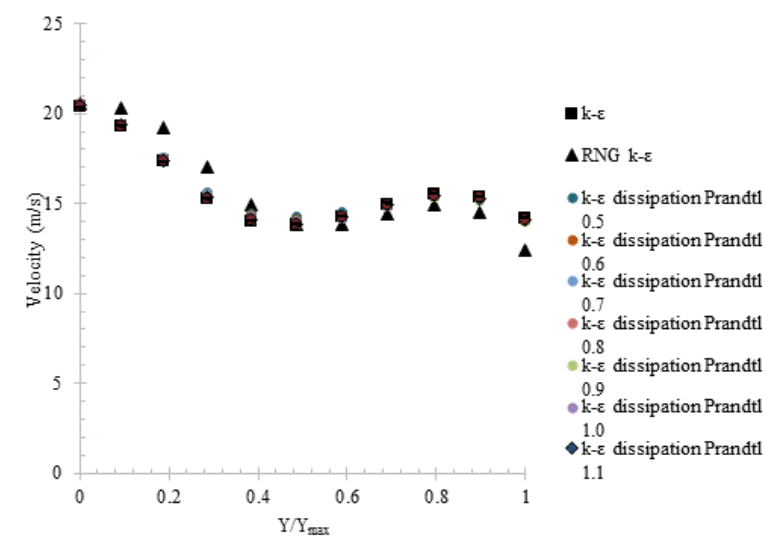

Fig. 12. Velocity distribution along the $y$-axis of the crossflow turbine blade

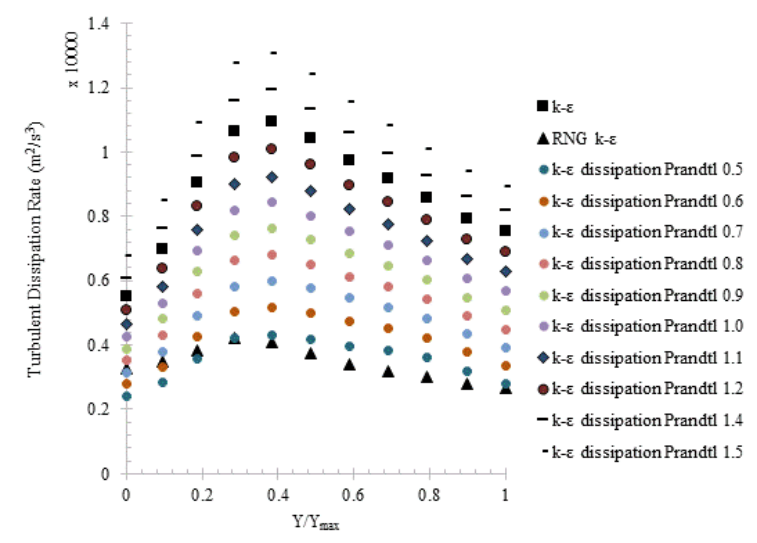

Fig. 14. Turbulent dissipation rate distribution along the $y$-axis of the crossflow turbine blade

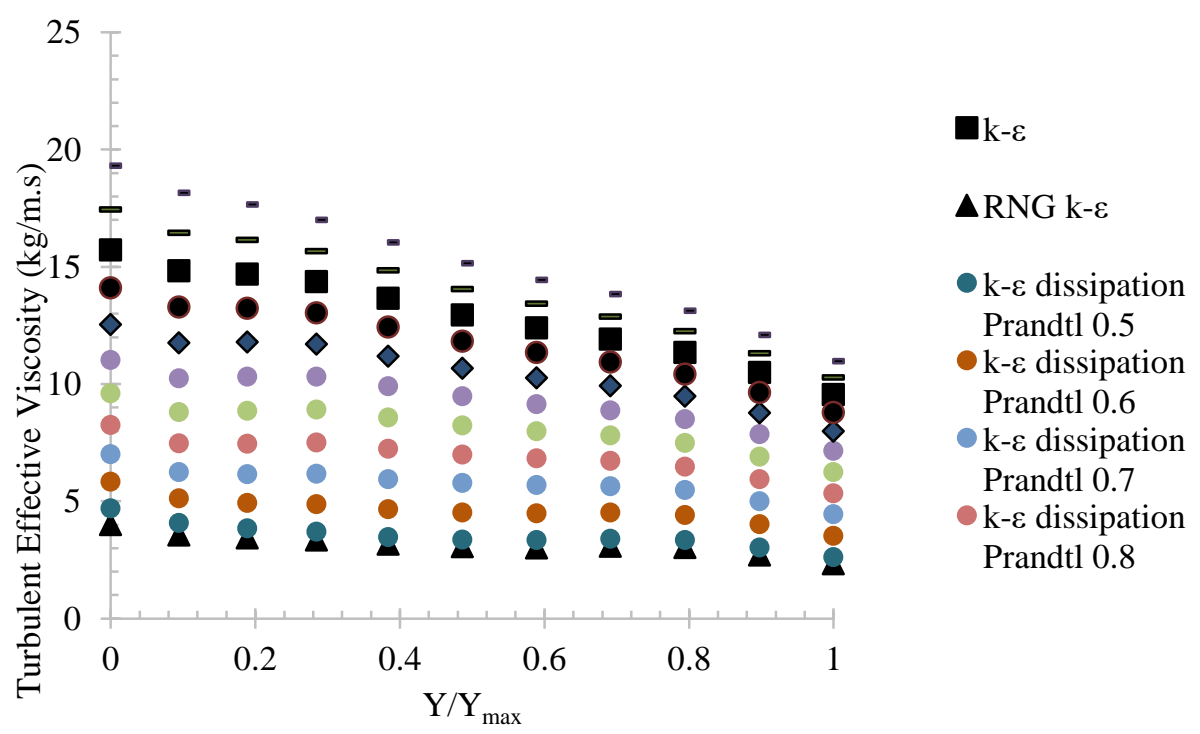

Fig. 15 Turbulent effective viscosity distribution along the $y$-axis of the crossflow turbine blade

For further investigation, adjustment of the Standard $k-\varepsilon$ model constants of $\sigma_{k}$ and $\sigma_{\varepsilon}$ using the Multi-Linear Regression (MLR) method is made on the center position of the first stage blade of 
the crossflow turbine. Shown in Table 2, the best average deviation of the MLR results against the results of the RNG k- $\varepsilon$ model on parameters; pressure at $\sigma_{\varepsilon}=0.8,0.9$ and $\sigma_{k}=1$, velocity at $\sigma_{\varepsilon}=0.8$ to 1.4 and $\sigma_{k}=1$, turbulent kinetic energy, turbulent dissipation rate, and turbulent effective viscosity at $\sigma_{\varepsilon}=0.5$ and $\sigma_{k}=1$. The range of average deviation in pressure and velocity parameters is quite small, while the range of mean deviation in the turbulence properties is tremendously large. These variables adjustments at the constant value of $\sigma_{k}=1$ are taken in the range $\sigma_{\varepsilon}=0.5$ to 1.5 . The analysis above shows that increasing the $\sigma_{\varepsilon}$ value will reduce the turbulent kinetic energy and its dissipation rate.

\section{Table 2}

MLR error of the calculation 1 for the RNG $k-\varepsilon$ model

\begin{tabular}{|c|c|c|c|c|c|c|c|}
\hline \multicolumn{3}{|c|}{$\begin{array}{l}\text { Optimization } \\
\text { Variable }\end{array}$} & \multicolumn{5}{|c|}{ MLR Calculation Error for The RNG k- $\varepsilon$ Model } \\
\hline No & $\sigma_{k}$ & $\sigma_{\varepsilon}$ & $\begin{array}{l}\text { Total } \\
\text { Pressure } \\
(\mathrm{Pa})\end{array}$ & $\begin{array}{l}\text { Velocity } \\
(\mathrm{m} / \mathrm{s})\end{array}$ & $\begin{array}{l}\text { Turbulent Kinetic } \\
\text { Energy }\left(\mathrm{m}^{2} / \mathrm{s}^{2}\right)\end{array}$ & $\begin{array}{l}\text { Turbulent } \\
\text { Dissipation Rate } \\
\left(\mathrm{m}^{2} / \mathrm{s}^{3}\right)\end{array}$ & $\begin{array}{l}\text { Turbulent Effective } \\
\text { Viscosity (kg/m.s) }\end{array}$ \\
\hline 1 & 1 & 0.5 & $7 \%$ & $3 \%$ & $8 \%$ & $12 \%$ & $10 \%$ \\
\hline 2 & 1 & 0.6 & $5 \%$ & $2 \%$ & $37 \%$ & $34 \%$ & $48 \%$ \\
\hline 3 & 1 & 0.7 & $2 \%$ & $1 \%$ & $66 \%$ & $55 \%$ & $89 \%$ \\
\hline 4 & 1 & 0.8 & $1 \%$ & $0 \%$ & $95 \%$ & $75 \%$ & $129 \%$ \\
\hline 5 & 1 & 0.9 & $1 \%$ & $0 \%$ & $123 \%$ & $95 \%$ & $169 \%$ \\
\hline 6 & 1 & 1.0 & $3 \%$ & $0 \%$ & $152 \%$ & $116 \%$ & $210 \%$ \\
\hline 7 & 1 & 1.1 & $7 \%$ & $0 \%$ & $179 \%$ & $136 \%$ & $249 \%$ \\
\hline 8 & 1 & 1.2 & $9 \%$ & $0 \%$ & $207 \%$ & $158 \%$ & $287 \%$ \\
\hline 9 & 1 & 1.3 & $11 \%$ & $0 \%$ & $235 \%$ & $180 \%$ & $324 \%$ \\
\hline 10 & 1 & 1.4 & $15 \%$ & $0 \%$ & $264 \%$ & $204 \%$ & $360 \%$ \\
\hline 11 & 1 & 1.5 & $17 \%$ & $1 \%$ & $295 \%$ & $233 \%$ & $395 \%$ \\
\hline
\end{tabular}

The results of the constant value modification which are taken in the range below $\sigma_{\varepsilon}=0.51$ and $\sigma_{k}=1$, are as shown in Table 3.

Table 3

MLR error of the calculation 2 for the RNG k- $\varepsilon$ model

\begin{tabular}{|c|c|c|c|c|c|c|c|}
\hline \multicolumn{3}{|c|}{$\begin{array}{l}\text { Optimization } \\
\text { Variable }\end{array}$} & \multicolumn{5}{|c|}{ MLR Calculation Error for The RNG k- $\varepsilon$ Model } \\
\hline No & $\sigma_{k}$ & $\sigma_{\varepsilon}$ & $\begin{array}{l}\text { Total } \\
\text { Pressure } \\
(\mathrm{Pa})\end{array}$ & $\begin{array}{l}\text { Velocity } \\
\text { (m/s) }\end{array}$ & $\begin{array}{l}\text { Turbulent Kinetic } \\
\text { Energy }\left(\mathrm{m}^{2} / \mathrm{s}^{2}\right)\end{array}$ & $\begin{array}{l}\text { Turbulent } \\
\text { Dissipation Rate } \\
\left(\mathrm{m}^{2} / \mathrm{s}^{3}\right)\end{array}$ & $\begin{array}{l}\text { Turbulent Effective } \\
\text { Viscosity }(\mathrm{kg} / \mathrm{m} . \mathrm{s})\end{array}$ \\
\hline 1 & 1 & 0.51 & $8 \%$ & $2 \%$ & $12 \%$ & $12 \%$ & $16 \%$ \\
\hline 2 & 1 & 0.5 & $8 \%$ & $2 \%$ & $9 \%$ & $10 \%$ & $12 \%$ \\
\hline 3 & 1 & 0.49 & $8 \%$ & $2 \%$ & $6 \%$ & $8 \%$ & $9 \%$ \\
\hline 4 & 1 & 0.48 & $8 \%$ & $2 \%$ & $3 \%$ & $6 \%$ & $5 \%$ \\
\hline 5 & 1 & 0.47 & $9 \%$ & $2 \%$ & $0 \%$ & $4 \%$ & $1 \%$ \\
\hline 6 & 1 & 0.46 & $9 \%$ & $2 \%$ & $2 \%$ & $2 \%$ & $3 \%$ \\
\hline 7 & 1 & 0.45 & $9 \%$ & $2 \%$ & $5 \%$ & $0 \%$ & $7 \%$ \\
\hline 8 & 1 & 0.44 & $9 \%$ & $2 \%$ & $8 \%$ & $3 \%$ & $11 \%$ \\
\hline 9 & 1 & 0.43 & $9 \%$ & $2 \%$ & $11 \%$ & $5 \%$ & $15 \%$ \\
\hline 10 & 1 & 0.42 & $10 \%$ & $2 \%$ & $14 \%$ & $7 \%$ & $18 \%$ \\
\hline
\end{tabular}

Modification of the $k-\varepsilon$ model with changes in the constant value $\sigma \varepsilon=0.47$ and $\sigma_{k}=1$ has an average deviation on physical parameters; pressure $9 \%$, velocity $2 \%$, as well as for turbulent parameters; turbulent kinetic energy $0 \%$, turbulent dissipation rate $4 \%$, and turbulent effective 
viscosity $1 \%$. Constant value $\sigma_{\varepsilon}=0.47$ and $\sigma_{k}=1$ are thus recommended to get a smaller deviation value compared to the k- $\varepsilon$ RNG model. As the result shows the increasing the $\sigma_{\varepsilon}$ value will reduce the turbulent kinetic energy and its dissipation rate as described above.

\section{Conclusion}

The numerical study has been carried out to study the effects of the $\sigma_{\varepsilon}$ value on the Standard $k-\varepsilon$ model performance for predicting recirculating flow in a crossflow turbine. The value of $\sigma_{\varepsilon}$ was varied from 0.5 to 1.5 in the CFD simulations, while the default value of the parameter is 1 . The assessment was also done using the Multi-Linear Regression (MLR) method with changes in the parameter value from 0.47 to 1.5 , to advance the analysis of the performance when the constant is set to be around 0.5. From both methods, it was obtained that the changes in $\sigma_{\varepsilon}$ can give rise to significant shifts in turbulence properties, such as turbulent kinetic energy, turbulent dissipation rate, and turbulent viscosity, which are important variables to determine the acoustic and vibration of the equipment. The best fit for the RNG $k-\varepsilon$ model was attained when $\sigma_{\varepsilon}=0.47$, implying that the decreasing $\sigma_{\varepsilon}$ will provide better results. However, the case is not applied to flow properties (pressure and velocity), wherein the deviations between the Standard and RNG model are notable. For the velocity field, there were no significant changes observed when the $\sigma_{\varepsilon}$ value was varied, as opposed to the turbulence properties. Further research must be performed to address the significance of the $\sigma_{\varepsilon}$ value on velocity and pressure fields as they are the commonly used variables for assessing performance. Nonetheless, the results from the present study have demonstrated the possibility to improve the performance of the Standard $k-\varepsilon$ model performance in predicting recirculating flow phenomenon by adjusting the constant dictating the dissipation rate of turbulent kinetic energy.

\section{Acknowledgment}

The authors would like to thank the Directorate of Research and Service Community (DRPM) Universitas Indonesia for funding this research with grant number NKB679/UN2.RST/HKP.05.00/2020 and to PT. CCIT Group Indonesia for CFDSOF ${ }^{\circledR}$ software license.

\section{References}

[1] Goldberg, U. C., D. K. Ota, and S. R. Chakravarthy. "Turbulence Modeling for Recirculating Flows-Reasons and Approach." In Instabilities and Turbulence in Engineering Flows, pp. 281-292. Springer, Dordrecht, 1993. https://doi.org/10.1007/978-94-011-1743-2 15

[2] Peláez Restrepo, Juan Diego. "Study of the effect of the geometrical parameters of the runner and operation conditions on performance and flow characteristics in a cross flow turbine." PhD diss., Universidad EAFIT, 2014.

[3] Reynolds, Osborne. "On the two manners of motion of water." In Proc. R. Instn Gt Brit, vol. 11, pp. 44-52. 1884.

[4] Adanta, Dendy, Budiarso Budiarso, and Ahmad Indra Siswantara. "Assessment of turbulence modelling for numerical simulations into pico hydro turbine." Journal of Advanced Research in Fluid Mechanics and Thermal Sciences 46, no. 1 (2018): 21-31.

[5] Darmawan, Steven, Ahmad Indra Siswantara, Budiarso, Asyari Daryus, Agus Tri Gunawan, Achmad Bayu Wijayanto, and Harto Tanujaya. "Turbulent Flow Analysis in Auxiliary Cross-Flow Runner of a Proto X-3 Bioenergy Micro Gas Turbine Using RNG k- $\varepsilon$ Turbulence Model." ARPN Journal of Engineering and Applied Sciences 10, no. 16 (2015): 7086-7091.

[6] Orszag, STEVEN A, and VICTOR Yakhot. "Renormalization Group Analysis of Turbulence." In Proceedings of the International Congress of Mathematicians, 1395-99, 1986.

[7] Yakhot, Victor, and Steven A. Orszag. "Renormalization group analysis of turbulence. I. Basic theory." Journal of scientific computing 1, no. 1 (1986): 3-51. https://doi.org/10.1007/BF01061452

[8] Yakhot, V. S. A. S. T. B. C. G., S. A. Orszag, Siva Thangam, T. B. Gatski, and CG1167781 Speziale. "Development of turbulence models for shear flows by a double expansion technique." Physics of Fluids A: Fluid Dynamics 4, no. 7 
(1992): 1510-1520. https://doi.org/10.1063/1.858424

[9] Hanjalic, K. "Closure models for incompressible turbulent flows." Lecture Notes at Von Kármán Institute 75 (2004).

[10] Sammartano, Vincenzo, Costanza Aricò, Armando Carravetta, Oreste Fecarotta, and Tullio Tucciarelli. "BankiMichell optimal design by computational fluid dynamics testing and hydrodynamic analysis." Energies 6, no. 5 (2013): 2362-2385. https://doi.org/10.3390/en6052362

[11] Gebrehiwot, Mekonnen Gebreslasie, Josse De Baerdemaeker, and Martine Baelmans. "Numerical Analysis of a Cross-Flow Fan with Two Outlets." In 5th International Conference on Heat Transfer, Fluid Mechanics and Thermodynamics. Sun City, 2007.

[12] Kim, Tae-An, D-W. Kim, S-K. Park, and Youn J. Kim. "Performance of a cross-flow fan with various shapes of a rearguider and an exit duct." Journal of mechanical science and technology 22, no. 10 (2008): 1876-1882. https://doi.org/10.1007/s12206-008-0726-9

[13] Cheng, Wee Teck. "Experimental and numerical analysis of a crossflow fan." Naval Postgraduate School, California, 2003.

[14] Sowa, A. N. D. R. Z. E. J. "Flow simulations in cross-flow fans using the finite element and finite volume methods." Task Quarterly 1 (2004).

[15] Choi, Young-Do, Jae-Ik Lim, You-Taek Kim, and Young-Ho Lee. "Performance and internal flow characteristics of a cross-flow hydro turbine by the shapes of nozzle and runner blade." Journal of fluid science and technology 3 , no. 3 (2008): 398-409. https://doi.org/10.1299/jfst.3.398

[16] De Andrade, Jesús, Christian Curiel, Frank Kenyery, Orlando Aguillón, Auristela Vásquez, and Miguel Asuaje. "Numerical investigation of the internal flow in a Banki turbine." International Journal of Rotating Machinery 2011 (2011). https://doi.org/10.1155/2011/841214

[17] Kim, I. C., Joji Wata, Mohammed R. Ahmed, and Y. H. Lee. "CFD study of a ducted cross flow turbine concept for high efficiency tidal current energy extraction." In Proceedings of Asian Wave and Tidal Energy Conference 2012, pp. 400-405. 2012.

[18] Qi-fei, Li, Quan Hui, and Li Ren-nian. "Influences of guide vanes airfoil on hydraulic turbine runner performance." Procedia Engineering 28 (2012): 703-708. https://doi.org/10.1016/j.proeng.2012.01.794

[19] Launder, Brian Edward, and Dudley Brian Spalding. "The numerical computation of turbulent flows." In Numerical prediction of flow, heat transfer, turbulence and combustion, pp. 96-116. Pergamon, 1983. https://doi.org/10.1016/B978-0-08-030937-8.50016-7

[20] Lakshminarayana, B. "Turbine cooling and heat transfer." Fluid Dynamics and Heat Transfer of Turbomachinery (1996): 597-721. https://doi.org/10.1002/9780470172629

[21] Marshall, Elizabeth Marden, and André Bakker. "Computational fluid mixing." Handbook of industrial mixing: science and practice (2004): 257-343. https://doi.org/10.1002/0471451452.ch5

[22] Choudhury, D. "Introduction to the renormalization group method and turbulence modeling (Technical Memorandum TM-107, Fluent Inc. 1993)." Google Scholar.

[23] Thangam, S., and Charles G. Speziale. "Turbulent flow past a backward-facing step-A critical evaluation of twoequation models." AIAA journal 30, no. 5 (1992): 1314-1320. https://doi.org/10.2514/3.11066

[24] AVVA, R. K., S. J. Kline, and J.H. Ferziger. "Technical Report TF-33." California, 1988.

[25] Sindir, Munir Mehmet Suat. A numerical study of turbulent flows in backward-facing step geometries: a comparison of four models of turbulence. University of California, Davis, 1982.

[26] Thangamftc, S. "Turbulent separated flow past a backward." (1991).

[27] Speziale, CharlesG. "On NonlinearK-I AndK-Modelsof Turbulence." J. Fluid Mech 178 (1987): 459-75. https://doi.org/10.1017/S0022112087001319

[28] Gunadi, Gun Gun R., Ahmad Indra Siswantara, Budiarso Budiarso, Hariyotejo Pujowidodo, Candra Damis Widiawaty, and Dendy Adanta. "Analysis of Inverse-Prandtl of Dissipation in Standard k-? Turbulence Model for Predicting Flow Field of Crossflow Wind Turbine." CFD Letters 12, no. 4 (2020): 68-78. https://doi.org/10.37934/cfdl.12.4.6878

[29] Orszag, Steven A. "Renormalisation group modelling and turbulence simulations." Near-wall turbulent flows (1993).

[30] Pujowidodo, Hariyotejo, Ahmad Indra Siswantara, Budiarso, Gun Gun R. Gunadi, and Asyari Daryus. "Turbulence model and validation of air flow in crossflow turbine nozzle." In AIP Conference Proceedings, vol. 2001, no. 1, p. 060006. AIP Publishing LLC, 2018. https://doi.org/10.1063/1.5050007

[31] Widiawaty, Candra Damis, Ahmad Indra Siswantara, Budiarso, Asyari Daryus, Gun Gun Ramdlan Gunadi, and Hariyotejo Pujowidodo. "Investigation the effect of superficial velocity to the heat transfer in bubbling regime of fluidization using CFD simulation." In AIP Conference Proceedings, vol. 2187, no. 1, p. 020024. AIP Publishing LLC, 2019. https://doi.org/10.1063/1.5138279

[32] Siswantara, A. I., H. Pujowidodo, M. A. Budiyanto, GG Ramdlan Gunadi, and C. D. Widiawaty. "An investigation turbulence model of standard $k-\varepsilon$ to get optimum parameters of turbulence constants $(c \mu, c 1 \varepsilon, a n d c 2 \varepsilon)$ of 
compressible fluid dynamics in a confined jet." Journal of Southwest Jiaotong University 56, no. 5 (2021). https://doi.org/10.35741/issn.0258-2724.56.5.26

[33] Roache, Patrick J. Verification and validation in computational science and engineering. Vol. 895. Albuquerque, NM: Hermosa, 1998. 